\title{
Fine structures on zero-field steps in low-loss Josephson tunnel junctions
}

\author{
Monaco, Roberto; Barbara, Paola; Mygind, Jesper
}

Published in:

Physical Review B

Link to article, DOI:

10.1103/PhysRevB.47.12292

Publication date:

1993

Document Version

Publisher's PDF, also known as Version of record

Link back to DTU Orbit

Citation (APA):

Monaco, R., Barbara, P., \& Mygind, J. (1993). Fine structures on zero-field steps in low-loss Josephson tunnel junctions. Physical Review B, 47(18), 12292-12295. https://doi.org/10.1103/PhysRevB.47.12292

\section{General rights}

Copyright and moral rights for the publications made accessible in the public portal are retained by the authors and/or other copyright owners and it is a condition of accessing publications that users recognise and abide by the legal requirements associated with these rights.

- Users may download and print one copy of any publication from the public portal for the purpose of private study or research.

- You may not further distribute the material or use it for any profit-making activity or commercial gain

- You may freely distribute the URL identifying the publication in the public portal 


\title{
Fine structures on zero-field steps in low-loss Josephson tunnel junctions
}

\author{
R. Monaco, ${ }^{*}$ P. Barbara, and J. Mygind \\ Physics Laboratory I, The Technical University of Denmark, DK-2800, Lyngby, Denmark
}

(Received 8 December 1992)

\begin{abstract}
The first zero-field step in the current-voltage characteristic of intermediate-length, high-quality, lowloss $\mathrm{Nb} / \mathrm{Al}-\mathrm{AlO} \mathrm{O}_{x} / \mathrm{Nb}$ Josephson tunnel junctions has been carefully investigated as a function of temperature. When decreasing the temperature, a number of structures develop in the form of regular and slightly hysteretic steps whose voltage position depends on the junction temperature and length. This phenomenon is interesting for the study of nonlinear dynamics and for application of long Josephson tunnel junctions as microwave and millimeter-wavelength oscillators.
\end{abstract}

The resonant motion of magnetic flux quanta (fluxons) in the barrier of a long (with respect to the Josephson penetration length $\lambda_{J}$ ) Josephson tunnel junction has been studied extensively in the last 20 years both by recording the dc current-voltage characteristic (IVC) of the zero-field steps (ZFS) and by detecting the radiation emitted as a result of fluxon reflections. Most of the experimental work reported in the literature was made on samples based on the native oxide of tin or niobium $\left(\mathrm{Sn} / \mathrm{SnO}_{x} / \mathrm{Sn}\right.$ and $\mathrm{Nb} / \mathrm{NbO}_{x} / \mathrm{Pb}$ junctions), which makes rather good tunneling barriers; however, by means of the present $\mathrm{Nb} / \mathrm{Al}-\mathrm{AlO}_{x} / \mathrm{Nb}$ technology based on a metal overlayer, very high-quality barriers can be realized which have almost negligible ohmic losses. The superior quality together with the improved thermal and mechanical stability of these new devices are being exploited for the practical utilization of superconducting tunnel junctions. Among the various applications, we presently focus on the use of a spatially extended Josephson tunnel junction in the resonant fluxon regime as a building block in microwave and millimeter-wavelength oscillators.

We report on a thorough experimental investigation of the properties of $\mathrm{Nb} / \mathrm{Al}-\mathrm{AlO}_{x} / \mathrm{Nb}$ intermediate-length Josephson tunnel junctions operated in the resonant fluxon regime, i.e., when dc current biased on a ZFS. We have limited our measurements to the first ZFS, which is related to the shuttling back and forth of a single fluxon; the fluxon dynamics is more complicated on higher-order steps. Most of our measurements were done by detecting the emitted radiation of the fundamental fluxon frequency $f$; this $\mathrm{rf}$ technique, because of the Josephson (reduced) voltage-frequency relation $V=2 \phi_{0} f \simeq 4$. 1 $\mu \mathrm{V} / \mathrm{GHz} \times f$ has, by far, a better resolution than its $\mathrm{dc}$ counterpart, i.e., the recording of the dc IVC.

In this paper, we focus our attention on the appearance of fine structures on the first zero-field step which occurs in high-quality samples and which is enhanced by lowering the operating temperature; after the presentation of the experimental results, we discuss the difference with previous related experiments.

The samples consisted of unidimensional overlap Josephson tunnel junctions, coupled to a microstrip line through a capacitive gap: The details of the geometry are shown in Fig. 3 of Ref. 1. The fabrication procedure is based on the anodization of the top layer of a $\mathrm{Nb} / \mathrm{Al}-\mathrm{AlO}_{x} / \mathrm{Nb}$ trilayer as described in Ref. 2 . As a result of this technique, the junction is embedded in a window opened in a $200-\mathrm{nm}$ thick $\mathrm{Nb}_{2} \mathrm{O}_{5}$ layer between the base electrode and the wiring layer, which are always larger than the junction itself. All the samples were fabricated in the Laboratory of Superconductivity at the University of Salerno, Italy.

The relevant parameters for the samples have been evaluated from the junction IVC, the magnetic pattern of the critical current, the voltage of the Fiske steps in small junctions, and the maximum frequency of the signal emitted by long junctions biased on the first ZFS. All tested junctions had the critical current density $J_{c}$ in the range 10-50 A/ $\mathrm{cm}^{2}$, specific capacitance $C_{s}=0.03 \mathrm{~F} / \mathrm{m}^{2}$, magnetic barrier thickness $d=160 \mathrm{~nm}$, and normalized lengths $l$ from 3 to 5 at $T=4.2 \mathrm{~K}$. We recall that, since the propagation velocity of electromagnetic waves in the junction barrier is $\bar{c}=\lambda_{j} \omega_{p}$ and the junction fundamental frequency of resonance is $f_{r}=\bar{c} / 2 L$ (which was within the microwave $X$ band for our samples), then the plasma frequency $\omega_{p} / 2 \pi$ can alternatively be obtained from the relation $2 \pi f_{r} / \omega_{p}=\pi / l$. The cryogenic setup and the microwave detection system have been described elsewhere. $^{3}$

Experiments on three samples gave qualitatively similar results and for the sake of simplicity, we will limit our attention to one particular sample whose geometrical and electrical (at $T=4.2 \mathrm{~K}$ ) parameters are reported in Table I and whose IVC (at $T=4.2 \mathrm{~K}$ ) is shown in Fig. 1. In Fig. 2 the stars are experimental data relative to the frequency $f$ of the emitted signal (at one end of the junction) vs the external bias current $I$ when the junction is biased on the first ZFS at $T=4.1 \mathrm{~K}$. The quasiparticle tunneling current is less than $10 \mu \mathrm{A}$ (one small division). It is evident that the curve is not continuous, but is split into a number of small isolated branches almost equally spaced in frequency. We remember that the fluxon average velocity $v$ is related to emitted signal frequency $f$ through $f=v / 2 L$, where $L$ is the junction length. The experiments show that the fluxon average velocity does not increase continuously with the energy externally supplied via the bias current, but jumps from one branch to the 
TABLE I. Geometrical and electrical (at $4.2 \mathrm{~K}$ ) parameters of the sample.

\begin{tabular}{ll}
\hline \hline & Data \\
Length $L$ & $500 \mu \mathrm{m}$ \\
Width $W$ & $20 \mu \mathrm{m}$ \\
Critical current $I_{c}$ & $1.15 \mathrm{~mA}$ \\
Critical current density $J_{c}$ & $11.5 \mathrm{~A} / \mathrm{cm}^{2}$ \\
Normal resistance $R_{n}$ & $1.4 \Omega$ \\
Subgap resistance $R_{\mathrm{sg}}$ (at $\left.2 \mathrm{mV}\right)$ & $28 \Omega$ \\
Gap voltage $V_{g}$ & $2.75 \mathrm{mV}$ \\
Quality factor $V_{m}$ & $32 \mathrm{mV}$ \\
Magnetic thickness $d$ & $160 \mathrm{~nm}$ \\
Josephson length $\lambda_{j}$ & $120 \mu \mathrm{m}$ \\
Normalized length $L / \lambda_{j}$ & 4 \\
Specific capacitance $C_{s}$ & $0.03 \mathrm{~F} / \mathrm{m}^{2}$ \\
Total capacitance $C$ & $300 \mathrm{pF}$ \\
Plasma frequency $f_{p}$ & $17 \mathrm{GHz}$ \\
Resonance frequency $f_{r}$ & $12.71 \mathrm{GHz}$ \\
$1 /\left(R_{\mathrm{sg}} C\right)$ frequency & $0.12 \mathrm{GHz}$ \\
McCumber $\beta_{c}$ & $8 \times 10^{5}$ \\
Swihart velocity $\bar{c}$ & $1.27 \times 10^{7} \mathrm{~m} / \mathrm{sec}$ \\
$\quad$ & \\
$\quad V_{m}=I_{c} R_{\text {sg }}, \quad \lambda_{j}=\sqrt{\hbar / 2 e \mu_{0} d J_{c}}, f_{p}$ & $=(1 / 2 \pi) \sqrt{2 e I_{c} / \hbar C}$ \\
$\quad \bar{c}=2 f_{r} L=2 \pi \lambda_{j} f_{p}, \beta_{c}=\left(2 \pi f_{p} R_{\mathrm{sg}} C\right)^{2}$ \\
\hline
\end{tabular}

next in such a way that certain values of the velocity are not allowed. Furthermore, sometimes the behavior is hysteretic and the current ranges of two contiguous branches overlap. When the hysteresis is very small (or absent) at the bias point where switching occurs two signals were observed simultaneously on the spectrum analyzer. This is due to the noise induced intermittence between the upper part of one branch and the lower part of the successive branch. The application of a small external magnetic field parallel to the plane of the junction and perpendicular to the long dimension of the junction tuned the frequency a few $\mathrm{MHz}$ upward independent of the field direction. However, the switching frequency positions did not change significatively.

The asymptotic resonance frequency in Fig. 2 is $f_{r}=12.69 \mathrm{GHz}$ so that, in normalized units, as indicated by the upper horizontal scale, the data only span a small frequency range from 0.91 to 1.00 . The positions of the

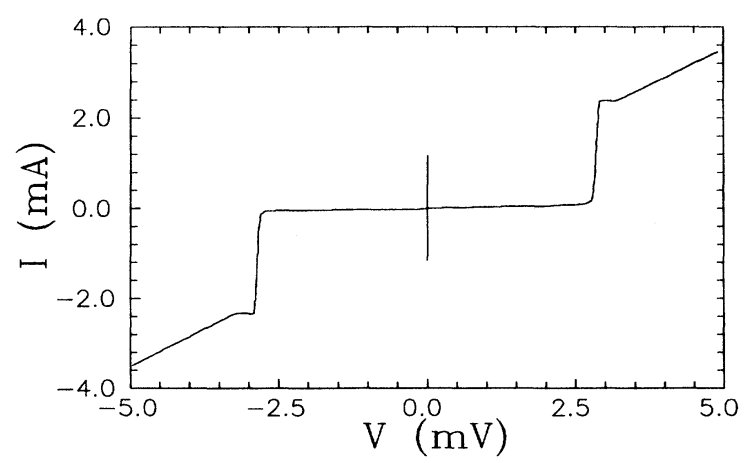

K.

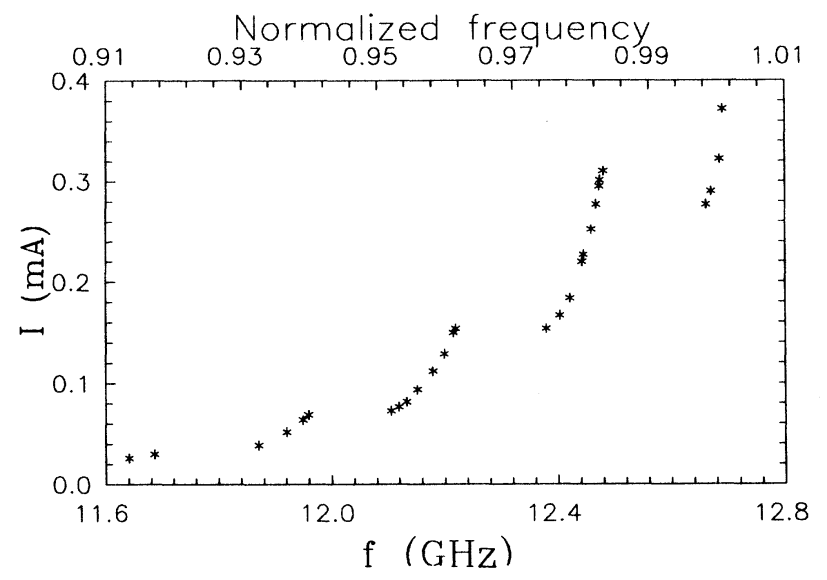

FIG. 2. First ZFS profile (emitted signal frequency vs external bias current) at $4.1 \mathrm{~K}$.

switching frequency for each branch are almost, but not exactly, equally spaced. (If we interpret these branches as resonances, the relatively large dynamical resistance of the lower structures suggests that the switching positions are somehow lower than the asymptotic resonant frequencies.) The distance between successive branches is very small; in normalized units it is less than $2 \%$ of the maximum frequency, and, for this sample corresponds to about $260 \mathrm{MHz}$ (the uncertainty of these frequency measurements is less than $1 \mathrm{MHz}$ ). With an effective voltage amplifier noise of $100 \mathrm{nV}$, the switching jumps from one branch to the next could be easily resolved in the dc IVC traced on the $X-Y$ recorder. The junction critical temperature was $T_{c}=8.9 \mathrm{~K}$ and the first ZFS was clearly visible, although very small, at about $T=8 \mathrm{~K}$. Figure 3 shows the evolution of the first ZFS for temperatures between $T=6.4 \mathrm{~K}$ [curve (a)] and $T=2.0 \mathrm{~K}$ [curve (g)]. For $T>6 \mathrm{~K}$ the ZFS profile is very smooth as in curve (a) whose upper part is practically linear. Decreasing the temperature results in a shift of the ZFS profile to higher

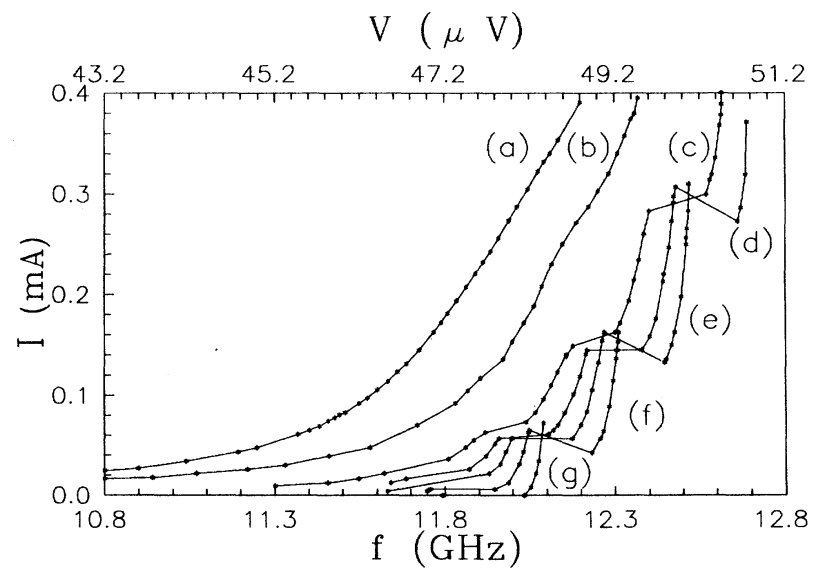

FIG. 3. First ZFS profile at different temperatures. (a) $6.4 \mathrm{~K}$, (b) $5.7 \mathrm{~K}$, (c) $4.5 \mathrm{~K}$, (d) $4.1 \mathrm{~K}$, (e) $3.9 \mathrm{~K}$, (f) $3.5 \mathrm{~K}$, and (g) $2.0 \mathrm{~K}$. The solid lines connecting the experimental points at each temperature are drawn as a guide to the eye. 
frequencies due to the change of the Swihart velocity $\bar{c}$ through the temperature dependence of the London penetration depths of the junction electrodes. For symmetrical junctions the dependence is $\bar{c}(T) / \bar{c}(0)=\left[1-\left(T / T_{c}\right)^{4}\right]^{1 / 4}$ being strongest for $T$ close to the critical temperature $T_{c}$. Lowering the temperature slightly below $6 \mathrm{~K}$, a number of superimposed bumps begin to develop in the upper part of the profile, as seen in curve (b); they gradually change into well defined not overlapping structures [curve (c)]. Note that the solid lines drawn between the experimental points obtained at the same temperature are unrelated to the switching to other branches. At even lower temperatures, these branches become more pronounced also in the lower part of the ZFS profile, as in curve (d) corresponding to Fig. 2. At the lowest temperatures the upper branches became unstable and they gradually disappear [curves (e), (f), and (g)] until at last the whole profile vanishes at temperatures below $T=2.0 \mathrm{~K}$. This is consistent with the dc measurements made by other authors ${ }^{4,5}$ where the amplitude of the ZFS decreases at low temperatures unlike other current singularities, which follow the critical current temperature dependence. Experimentally, since in highquality junctions and, especially, at low temperatures it becomes increasingly difficult to latch onto any low voltage current singularity, the measurements illustrated in Figs. 2 and 3 were obtained by first raising the temperature to a value where it was possible to latch onto the singularity, then varying the current, choosing an appropriate bias point, and finally lowering the temperature to the desired value. Similar measurements made on other two samples having a smaller length ( $L=350 \mu \mathrm{m}$ instead of $L=500 \mu \mathrm{m}$ ) showed very similar behavior, the main difference being a lower frequency separation between successive branches (scaling proportionally from about 170 to about $260 \mathrm{MHz}$ ). The same results have been obtained after repeated thermal cycles and under a number of experimental conditions, i.e., by changing the sampleholder, the mounting of the junctions (with and without ground plane), and the rf coupling to the external waveguide and coaxial cable. Furthermore, we have carried out a series of measurements in order to exclude the existence of an on-chip spurious resonance, both at low (VHF and UHF) and high ( $X$ band) frequencies. From this we infer that the splitting of the ZFS into many branches is due to internal mechanisms. This is further supported by the consideration that the main effect of the lowering of the temperature is a strong decrease of the shunt loss of the barrier; this is particularly true in the range $0<T<T_{c} / 2$ in which other relevant quantities such as the Josephson current and the London penetration depths remain virtually unchanged. In contrast, the losses due to the dissipation in the barrier, which are usually referred to in the literature by the dimensionless frequency independent damping parameter $\alpha=1 / \sqrt{\beta_{c}}$, have a strong temperature dependence, ${ }^{6}$ since they are related to the subgap resistance $R_{\mathrm{sg}}$, that is, to the tunneling of quasiparticles across the barrier (see the legend of Table I). Recently, it has been demonstrated ${ }^{2,7,8}$ that the subgap current of high-quality $\mathrm{Nb} / \mathrm{Al}-\mathrm{AlO}_{x} / \mathrm{Nb}$ superconducting tunnel junctions follows very closely an exponential dependence down to temperatures below 1.5 $\mathrm{K}$, in particular for small voltage values $(<500 \mu \mathrm{V})$. For the sample described here, the dependence of the subgap current on the temperature is shown in Fig. 4 in which a logarithmic scale is needed to evidence the strong temperature dependence typical of high-quality superconducting tunnel junctions. These data were taken by applying a small magnetic field $(\simeq 0.1 \mathrm{~T})$ in order to suppress the Josephson current and the Fiske resonances.

Curve (a) refers to the normal sample (at $T>T_{c}$ ) and corresponds to an ohmic curve with $R_{n}=0.14 \Omega$. Inserting the experimental value of the subgap resistance measured at, say, $V=500 \mu \mathrm{V}, \alpha$ goes from $4 \times 10^{-3}$ at $T=6.4 \mathrm{~K}$ [curve (b) of Fig. 4] to $2 \times 10^{-4}$ at $T=2.0 \mathrm{~K}$ [curve (f) of Fig. 4]. On the contrary, the frequency dependent losses due to the surface impedance of the superconducting junction electrode are only weakly temperature dependent.

It is well known that in underdamped unidimensional Josephson tunnel junction richer nonlinear phenomena occur due to the intimate interaction of the fluxons with persistent oscillations along the line originated by the fluxons themselves ${ }^{9,10}$ (the normalized time over which these oscillations decay goes as $1 / \alpha$ ). This idea has been used to explain the regular fine structures observed on the first ZFS of very long $\mathrm{Sn} / \mathrm{SnO}_{x} / \mathrm{Sn}$ junctions. ${ }^{11,12}$ In particular, in Ref. 11 the fine structures were related to the resonant interaction of $n$ fluxon oscillations with the $m$ th fractional cavity mode determined by $f_{r}=\bar{c} / L$ assuming the response of the Josephson resonator to be linear: The resonant frequencies in that case were found to correspond to $f_{n / m}=(n / m) f_{r}$ where $n$ and $m$ can be any integer numbers. Although supported by numerical simulations, ${ }^{13}$ this scheme is questionable; in fact, later, taking into account the nonlinear nature of a Josephson cavity, regular fine structures observed on similar junctions were interpreted as due to the interaction of the traveling fluxon with small amplitude plasma oscillations at $f_{p}$, i.e., with $k=0,{ }^{12}$ so that the resistive branches occur at $f_{n}=f_{p} / n$. [It is well known that the dispersion relation of small amplitude plasma oscillations and in the limit of zero bias is $\omega^{2}=\bar{c}^{2} k^{2}+\omega_{p}^{2}$, which for $k=0$ gives the

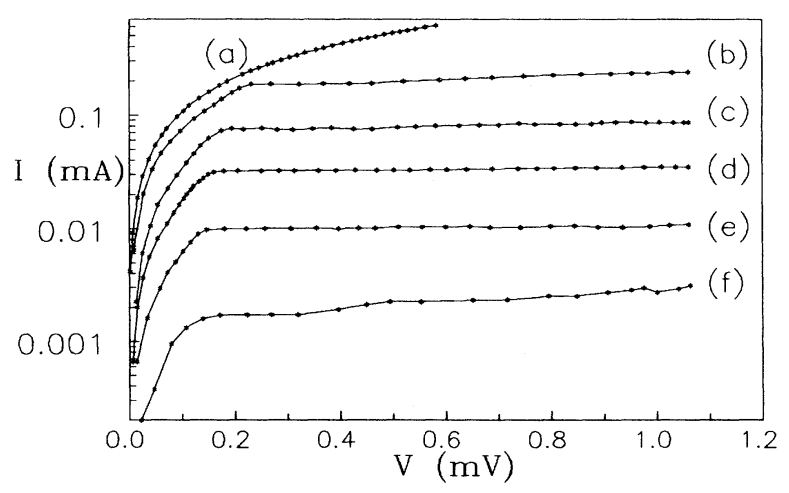

FIG. 4. Subgap current at different temperatures. The temperatures are (a) $10 \mathrm{~K}$, (b) $6.4 \mathrm{~K}$, (c) $4.6 \mathrm{~K}$, (d) $3.6 \mathrm{~K}$, (e) $2.8 \mathrm{~K}$, (f) $2.0 \mathrm{~K}$. The solid lines connecting the experimental points at each temperatures are drawn as a guide to the eye. 
lower cutoff frequency $\omega=\omega_{p}$; in underdamped junctions the approximation of small amplitudes has to be removed and the dispersion relation $\omega(k)$ becomes very complicated, since it involves complete elliptic integrals ${ }^{14}$.] However the restrictions to small amplitude, bias independent, and $k=0$ plasma oscillations do not have any physical justification but it is surprising that in our data the resonant frequencies $f_{r}$ can be rational related to a frequency $f^{*}$ by $f_{r}=n / m f^{*}$, where $n$ and $m$ are integer numbers and $f^{*}$ is very close to the plasma frequency; for example, for a particular sample, we found that the branches shown in Fig. 2 are tentatively related to the series $\frac{12}{18}, \frac{13}{19}, \frac{14}{20}, \frac{15}{21}, \frac{16}{22}$ with $f^{*}=17.5 \mathrm{GHz}$; analogous series can be found also for the other samples.

We wish to stress that a phenomenological model without restrictive conditions is very difficult to rule out so that resorting to numerical simulations is mandatory. However, great care must be used to simulate low damping nonlinear systems, since, besides the longer transients, the results are very sensitive to the numerical algorithm adopted to integrate the partial differential equation. ${ }^{15}$ We do not exclude that fine structures similar to those reported here have already been observed on high-quality long Josephson tunnel junctions although interpreted in terms of different mechanisms (see, for example, Ref. 16).

In conclusion, the observed regular fine structures on the ZFS of high-quality Josephson junctions is a phenomenon which deserves further investigation both motivated by its interest in the basic study of nonlinear dynamic systems and also to better ascertain the feasibility of long Josephson tunnel junctions as practical devices.

We are pleased to acknowledge fruitful discussions with G. Costabile, R. D. Parmentier, N. F. Pedersen, and A. Ustinov. One of the authors (P.B.) gratefully acknowledges the University of Salerno (Italy) for financial support. This work was partially supported by the National Research Council of Italy under the Progetto Finalizzato "Superconductive and Cryogenic Technology."
*Permanent address: Istituto di Cibernetica del CNR, I-80072 Arco Felice, Italy.

${ }^{1}$ G. Costabile, R. Monaco, and S. Pagano, J. Appl. Phys. 63, 5406 (1988).

${ }^{2}$ R. Monaco, R. Cristiano, L. Frunzio, and C. Nappi, J. Appl. Phys. 71, 1888 (1992).

${ }^{3}$ E. Joergensen, V. P. Koshelets, R. Monaco, J. Mygind, M. R. Samuelsen, and M. Salerno, Phys. Rev. Lett. 49, 1093 (1982).

${ }^{4}$ A. M. Cucolo, R. Monaco, S. Pace, R. D. Parmentier, B. Savo, and R. Vaglio, Physica B\&C 107B, 547 (1981).

${ }^{5}$ T. V. Rajeevakumar, J. X. Przybysz, J. T. Chen, and D. N. Langenberg, Phys. Rev. B 22, 2392 (1980).

${ }^{6}$ N. F. Pedersen and D. Welner, Phys. Rev. B 29, 2551 (1984).

${ }^{7}$ E. C. G. Kirk, M. G. Blamire, R. E. Somekh, and J. E. Evetts, IEEE Trans. Magn. MAG-27, 3137 (1991).

${ }^{8}$ D. J. Adelerhof, E. P. Houwman, P. B. M. Fransen, D. Veldhuis, J. Flokstra, and H. Rogalla, IEEE Trans. Magn.
MAG-27, 3153 (1991).

${ }^{9}$ A. Davidson, N. F. Pedersen, and S. Pagano, Appl. Phys. Lett. 48, 1306 (1986).

${ }^{10}$ S. Pagano, M. P. Sorensen, P. L. Christiansen, and R. D. Parmentier, Phys. Rev. B 38, 4677 (1988).

${ }^{11}$ M. R. Scheuermann, T. V. Rajeevakumar, J. J. Chang, and J. T. Chen, Physica B\&C 107B, 543 (1981).

${ }^{12}$ M. R. Scheuermann, C. C. Chi, N. F. Pedersen, J. J. Chang, and J. T. Chen, Appl. Phys. Lett. 48, 189 (1986).

${ }^{13}$ J. J. Chang, J. T. Chen, M. R. Scheuermann, and D. J. Scalapino, Phys. Rev. B 31, 1658 (1985).

${ }^{14}$ G. Costabile, R. D. Parmentier, B. Savo, D. W. McLaughlin, and A. C. Scott, Appl. Phys. Lett. 32, 587 (1978).

${ }^{15}$ R. D. Parmentier and G. Rotoli (private communication).

16J. Holm, J. Mygind, N. F. Pedersen, P. Barbara, G. Filatrella, and A. Davidson (unpublished). 